\title{
Enumeration and Alertness in Developmental Dyscalculia
}

\section{Supplemental Materials}

\section{MATAL diagnosis - developmental dyscalculia (DD)}

Table 1: Results of subtests in the MATAL diagnosis of DD participants.

\begin{tabular}{|c|c|c|}
\hline & Subtest & DD scor \\
\hline \multicolumn{3}{|l|}{ Arithmetic } \\
\hline & Retrieval of arithmetic facts RT & $-4.3(1)$ \\
\hline & Retrieval of arithmetic facts ACC & $-4.1(2)$ \\
\hline & Procedural knowledge RT & $-2.3(1)$ \\
\hline & Procedural knowledge ACC & $-4.3(1)$ \\
\hline & Number estimation RT & $-2.3(1)$ \\
\hline & Number estimation ACC & $-2.7(1)$ \\
\hline \multicolumn{3}{|l|}{ Reading } \\
\hline & Vocal text reading $\mathrm{RT}$ & $.3(1)$ \\
\hline & Vocal text reading ACC & $.1(.8)$ \\
\hline & Phonemic deletion RT & $0(.7)$ \\
\hline & Phonemic deletion ACC & $0(.6)$ \\
\hline & Phonemic counting RT & $.1(1)$ \\
\hline & Phonemic counting ACC & $.2(.6)$ \\
\hline & Non-word reading $\mathrm{RT}$ & $-.3(.7)$ \\
\hline & Non-word reading ACC & $-.4(.8)$ \\
\hline & Rapid automatic naming (objects) & $-.2(1)$ \\
\hline
\end{tabular}

Attention

CPT omitting 
Note. Scores are reported as Z-scores (mean $=0, S D=1$ ); standard deviation in parentheses $; \mathrm{CPT}=$ Continuous Performance Test $; \mathrm{RT}=$ reaction time; $\mathrm{ACC}=$ accuracy; ANT $=$ Attention Network Test. 


\section{Matching DD participants to controls - Arithmetic Battery and Additional Tests}

Part 1: Number comprehension and production

a. Comparing digits. Comparison of eight pairs of numbers: two pairs of 3-digit numbers, three pairs of 4-digit numbers and three pairs of 5-digit numbers. The participants had to mark a circle on the largest number.

b. Counting forward and backward. The participant had to count forward seven times (e.g., count from 793-802) and backward four times (e.g., count from 813798). The initial number was a 2-, 3- or 4-digit number.

c. Serial order. Participants were presented with 14 arithmetical series and had to fill the three numbers in the sequence, from largest to smallest and from smallest to largest.

d. Comparing fractions. The participants were presented with eight pairs of decimals and had to circle the largest number.

e. Verbal problems. Eight verbal problems were presented and participants were asked to: 1) Circle the correct operation for the given problem (addition, subtraction, multiplication or division). 2) Write down the representative equation. 3) Solve the equation. For example: In a building, there are 31 floors. The height of each floor is 4 meters. What is the height of the building? Answer: step 1 - the correct operation is multiplication; step 2 - the correct equation is: $31 \times 4=$; and step 3 - the correct answer is: 124.

f. Procedural knowledge. Participants were presented with 8 horizontal equations and were asked to copy them to vertical equations. Four equations were composed of real numbers and four equations were composed of decimals. The real number equations included two, three or four numbers of 1-, 2-, 3- or 4-digit numbers. For example $21,306+28+2+236=$ for the real numbers and $3.27+0.365=$ for the decimal equations. 


\section{Part 2 - Calculation}

a. Simple pure operations. Single-digit operations were administered (10 additions, 10 subtractions, 10 divisions and 10 multiplications). Each kind of operation appeared separately.

b. Simple mixed operations. Single-digit operations were administered (five additions, five subtractions, five divisions and five multiplications), mixed in one block in a random order. The equations had the same level of difficulty as in the pure block.

c. Decimal. There were four complex addition and four complex subtraction exercises requiring knowledge of decimals. The exercises were presented vertically. The numbers contained two digits after the decimal point.

d. Estimation. The experimenter asked participants to estimate the results of an equation (four additions, four subtractions, four divisions and four multiplications), or to decide whether the result would be bigger or smaller than a specific number. For example, estimate the result of $589+426$.

e. Comparing equations. Participants were asked to decide which equation would result larger number. Participants were instructed to decide according to estimation and not accurate calculation. There were eight comparisons (two additions, two subtractions, two divisions and two multiplications). For example, $71-38$ or $73-28$.

f. Vertical operation. Participants had to solve complex written arithmetic problems (addition, subtraction, multiplication, and division). The first eight exercises were addition and subtraction, and the remaining eight were multiplication and division. Exercises included problems with and without borrowing using each operation. 
Additional tests - Controls and DD participants

Table 2: IQ, Attention and reading tests.

\begin{tabular}{lcccc}
\hline & Controls & DD & $\boldsymbol{t}$ & $\boldsymbol{p}$ \\
\hline Raven (correct answers, out of 60) & $47.9(4.9)$ & $47.4(6.5)$ & .22 & .82 \\
CPT (RT) & $.11(11)$ & $.12(19)$ & -1.1 & .28 \\
CPT (ACC) & $94(3.8)$ & $94(3.6)$ & -.9 & .36 \\
Reading test (words per minute) & $127(13)$ & $126(17)$ & .26 & .8 \\
Reading test (ACC) & $100(0.4)$ & $99(0.7)$ & 1.2 & .23 \\
Non-Word (RT) & $36(13)$ & $41(16)$ & .42 & .68 \\
Non-Word (ACC) & $95(13)$ & $85(16)$ & 1.78 & .09
\end{tabular}

Note. Standard deviations in parentheses; $\mathrm{CPT}=$ Continuous Performance Test; RT $=$ reaction time; $\mathrm{ACC}=$ accuracy. 
Table 3: Arithmetic battery Part A: Number comprehension and production.

\begin{tabular}{|c|c|c|c|c|}
\hline & Controls & DD & $t$ & $p$ \\
\hline Comparing digits (RT) & $11(2.7)$ & $24(8.3)$ & -5.5 & $<.001$ \\
\hline Comparing digits (ACC) & $99(3.2)$ & $99(3.2)$ & .05 & .96 \\
\hline Counting forward (RT) & $52(10)$ & $83(28)$ & -3.9 & $<.001$ \\
\hline Counting forward (ACC) & $88(9)$ & $81(11)$ & 1.77 & .08 \\
\hline Counting backwards (RT) & $49(12)$ & $76(19)$ & -4.54 & $<.001$ \\
\hline Counting backwards (ACC) & $92(12)$ & $75(17)$ & 3.09 & .004 \\
\hline Serial order (RT) & $85(34)$ & $318(5)$ & -25.5 & $<.001$ \\
\hline Serial order (ACC) & $97(5)$ & $91(9)$ & 2.16 & .039 \\
\hline Comparing fractions (RT) & $12(3)$ & $40(18)$ & -5.89 & $<.001$ \\
\hline Comparing fractions (ACC) & $98(10)$ & $80(31)$ & 2.02 & .05 \\
\hline Verbal problems (RT) & $201(78)$ & $392(146)$ & -4.4 & $<.001$ \\
\hline Verbal problems (ACC) & $85(8)$ & $54(19)$ & 5.73 & $<.001$ \\
\hline Procedural knowledge (RT) & $159(56)$ & $261(145)$ & -2.58 & .016 \\
\hline Procedural knowledge (ACC) & $84(13)$ & $47(22)$ & 5.5 & $<.001$ \\
\hline
\end{tabular}

Note. Standard deviations in parentheses; $\mathrm{RT}=$ reaction time; $\mathrm{ACC}=$ accuracy. 
Tabl

\begin{tabular}{|c|c|c|c|c|}
\hline & Control & DD & $t$ & $p$ \\
\hline \multicolumn{5}{|l|}{ Simple Operations } \\
\hline Addition (RT) & $15(6)$ & $34(13)$ & -5.2 & $<.001$ \\
\hline Addition (ACC) & $99(3)$ & $94(13)$ & 1.15 & .27 \\
\hline Subtraction (RT) & $11(5)$ & $33(14)$ & -5.6 & $<.001$ \\
\hline Subtraction (ACC) & $99(2)$ & $98(4)$ & 1.06 & .3 \\
\hline Multiplication (RT) & $16(7)$ & $102(79)$ & -4.22 & .001 \\
\hline Multiplication (ACC) & $98(5)$ & $77(21)$ & 3.79 & .002 \\
\hline Division (RT) & $18(11)$ & $99(92)$ & -3.38 & .002 \\
\hline Division (ACC) & $96(5)$ & $67(28)$ & 3.9 & .001 \\
\hline Mixed operations (RT) & $58(31)$ & $212(149)$ & -3.95 & $<.001$ \\
\hline Mixed operations (ACC) & $92(10)$ & $71(19)$ & 3.8 & .001 \\
\hline Decimals (RT) & $72(37)$ & $123(53)$ & -3.07 & .005 \\
\hline Decimals (ACC) & $88(13)$ & $58(33)$ & 3.28 & .004 \\
\hline Estimation (ACC) & 77 (13) & 45 (19) & 5.35 & $<.001$ \\
\hline Comparing equations (RT) & $42(13)$ & $104(20)$ & -9.76 & $<.001$ \\
\hline Comparing equations (ACC) & $90(10)$ & $82(17)$ & 1.47 & .16 \\
\hline Vertical operation (RT) & $56(18)$ & $141(53)$ & -5.89 & $<.001$ \\
\hline Vertical operation (ACC) & $100(0)$ & $91(18)$ & 1.78 & .1 \\
\hline
\end{tabular}

; $\mathrm{ACC}=$ accuracy. 